\title{
Short communication: Correlation between within-herd antibody- prevalence and bulk tank milk antibody levels to Mycobacterium avium ssp. paratuberculosis using 2 commercial immunoassays
}

\author{
M. N. Pesqueira, ${ }^{*}$ E. Yus,† C. Factor, ${ }^{*}$ I. Mato, ${ }^{*}$ M. L. Sanjuán,† C. Eiras, $\ddagger$ I. Arnaiz, $\ddagger$ and F. J. Diéguez $\ddagger \S^{1}$ \\ *Sanitary Defense Group (ADSG) Xundeva, Polígono Industrial Lalín 2000, Parcela B2/B3, 36500 Lalín, Spain \\ †Institute of Food Analysis and Research (Animal Health and Epidemiology Unit), Veterinary Faculty of Lugo, Santiago de Compostela University, \\ Campus Universitario, 27002 Lugo, Spain \\ $\ddagger$ Animal Health and Production Laboratory of Galicia, Av. Madrid 77, 27002 Lugo, Spain \\ $\S A n a t o m y$ and Animal Production, and Veterinary Sciences Department, Veterinary Faculty of Lugo, Santiago de Compostela University, \\ Campus Universitario, 27002 Lugo, Spain
}

\begin{abstract}
The objective of this study was to determine the correlation between the results obtained with the ELISA technique for antibodies to Mycobacterium avium ssp. paratuberculosis in serum and bulk tank milk at the herd level. For this purpose, 203 samples of bulk tank milk were analyzed with 2 commercial ELISA from dairy herds with a prevalence of seropositive animals that was also determined. In regard to the reference test (results in blood serum), the sensitivity of the bulk tank milk test to detect high-positive herds ( $\geq 10 \%$ seroprevalence) ranged from 85.7 to $71.4 \%$. The specificity to detect herds with no seropositive animals ranged from 70.5 to $53 \%$. In a quantitative approach, Pearson correlation coefficients, reported as a measure of the linear association between herd seroprevalences and transformed optical density values recorded in bulk tank milk, were 0.39 and 0.54 for the studied ELISA. Although the test results were relatively fairly correlated with the within-herd prevalence, the practical utility of bulk tank milk testing for Mycobacterium avium ssp. paratuberculosis seems limited, especially regarding specificity.
\end{abstract}

Key words: dairy cattle, milk, serology, Johne's disease

\section{Short Communication}

Mycobacterium avium ssp. paratuberculosis (MAP) is the causal agent of paratuberculosis (Johne's disease), a chronic enteric granulomatous disease in ruminants; the disease seems to respond to antibiotics as symp-

Received February 10, 2017.

Accepted May 24, 2017.

${ }^{1}$ Corresponding author: franciscojavier.dieguez@usc.es toms weaken, but recur after antibiotics are no longer administered. Testing options for determination of herd MAP status include, mainly, individual or pooled fecal culture, culture of environmental samples, and ELISA on milk or serum (Lavers et al., 2014). Although the relative sensitivity (Se) of ELISA for detection of antibodies against MAP as compared with fecal culture is rather low, ELISA technology has gained an important place in herd-based testing schemes because of its low cost, fast processing time, and high-throughput potential (van Weering et al., 2007). Antibody ELISA is the most commonly used laboratory test for the detection of specific antibodies against MAP in serum and milk (Khol et al., 2013).

The adaptation of the ELISA technique for detecting antibodies in bulk tank milk (BTM) samples constitutes an inexpensive test, as it provides information about the status of a large group of animals from a single sample. Bulk tank milk testing is a frequently used tool for the surveillance and monitoring of several infectious diseases in dairy cattle, such as infectious bovine rhinotracheitis (IBR), enzootic bovine leucosis, bovine virus diarrhea (BVD), and salmonellosis. Bulk tank milk for BVD, IBR, and salmonellosis has been widely used to estimate the within-herd prevalence of antibody-positive cows and to monitor dairy herds (Nylen et al., 1999; Warnick et al., 2006; Diéguez et al., 2008; Eiras et al., 2012).

The utilization of BTM samples could also be a practical and cost-effective way to screen dairy herds for the presence of MAP. Milk sampling is markedly more convenient than fecal or blood sample testing for any disease of dairy cattle (Wilson et al., 2010). However, literature is scarce about the correlation between the BTM response and the within-herd prevalence for MAP infections.

The Se and specificity (Sp) of antibody ELISA against MAP vary widely, depending on the product 
and gold standard applied (Khol et al., 2013). When used on BTM, the ELISA tests have been shown to perform similarly to serum ELISA assays at the herd level, with an Se of 56 to $83 \%$ when fecal culture is used as a reference (Lombard et al., 2006; Wilson et al., 2010). The objective of our study was to examine the correlation between within-herd seroprevalences and BTM antibody levels against MAP in dairy cattle using 2 commercial immunoassays.

The study was performed in northwestern Spain, which is the major cattle-farming region of the country and the ninth within the European Union, accounting for $38 \%$ of milk production in Spain and $1.5 \%$ in the European Union (MAPAMA, 2015). Galicia was the first region in Spain to establish a voluntary MAP control program, which began in 2004 and remains active. The percentage of Galician herds involved increased from $4.6 \%$ in 2004 to $45 \%$ in 2013 . Vaccination against MAP is not permitted, as the region is not free of tuberculosis.

To evaluate the correlation between the levels of antibodies in a sample of BTM and the within-herd prevalence, 203 samples of BTM were collected from 203 dairy herds taking part in the voluntary control program. The seroprevalence in each herd was also determined. For this purpose, serum from every animal that was lactating at the time of serum collection was gathered less than $1 \mathrm{wk}$ before BTM collection. Blood samples were obtained by tail vein venipuncture using Venoject tubes (Terumo Europe, Leuven, Belgium) without anticoagulant.

The milk samples were skimmed by centrifugation $(3,000 \times g, 20 \mathrm{~min}$, room temperature $)$ and frozen at $-21^{\circ} \mathrm{C}$. The serum samples were frozen after blood clotting and stored with milk samples until tested. The samples were tested for antibodies against MAP with 2 commercial available indirect ELISA. The ELISA were designated as ELISA A (Idexx Paratuberculosis Screening Ab Test, Westbrook, ME; based in technology of Pourquier Institute) and ELISA B (Mycobacterium paratuberculosis test kit Parachek 2, Prionics AG, Zurich, Switzerland). All analyses were performed by following the recommendations of the manufacturer. All samples were preabsorbed with sonicates of environmental Mycobacterium phlei.

For both ELISA, optical density (OD) values were expressed as sample-to-positive ratio $(\mathrm{S} / \mathrm{P})$, calculated as follows:

(OD value of the sample - the OD value of the negative control)/(OD value of the positive control

- the OD value of the negative control) $\times 100$.
Serum samples were considered positive at an $\mathrm{S} / \mathrm{P}$ ratio greater than or equal to 55 (ELISA A) or 15 (ELISA B).

For analysis, herds were initially categorized based on the detected seroprevalence of MAP-specific antibodies as negative ( $0 \%$ within-herd seroprevalence), positive $(>0-<10 \%)$, and high positive $(\geq 10 \%)$. To estimate the accuracy of BTM samples to correctly classify negative, positive, and high-positive herds, threshold values of transformed OD were calculated for each of the mentioned groups for both ELISA. For this purpose, the mean values of transformed OD resulting from analysis of BTM samples were estimated for each of the 3 seroprevalence groups. From these estimated mean values and their corresponding confidence interval, threshold values that allow the prediction of herd seroprevalence status from BTM were established. This was carried out by simple (linear) interpolation, as the straight line between the upper limit of the confidence interval of one prevalence group and the lower limit of the next one.

The Se of the BTM test with respect to the serum samples serving as reference was evaluated in terms of the proportion of positive and high-positive herds which the BTM test classified as positive and highly positive, respectively. Specificity was evaluated in terms of the proportion of negative herds ( $0 \%$ seropositive animals), which the BTM test classified as negative. In addition, the correlation between within-herd seroprevalence and antibody levels in BTM was evaluated in a quantitative approach, using the Pearson correlation coefficients $(\rho)$. All analysis was done with SPSS version 12.0 (SPSS Inc., Chicago, IL).

The mean herd size of the surveyed herds was 51.1 cows ( $\mathrm{SD}=33.4$; 45.5 for negative herds; 64.1 for positive herds and 55.2 for highly positive herds). Of the 203 farms used in the study, 132 had $0 \%$ seroprevalence, 57 had $>0$ to $<10 \%$ seropositive animals, and 14 had $\geq 10 \%$. The mean values of transformed OD resulting from analysis of BTM samples for each of the seroprevalence groups are summarized in Table 1.

The cut-off points, calculated to establish the intervals of transformed OD values that enabled classification of a herd within a corresponding seroprevalence group from a BTM sample, are summarized in Table 2 . Using these cut-off points, with respect the reference test (results in blood serum), the Se of BTM ELISA to correctly classify high-positive herds $(\geq 10 \%$ seroprevalence) was $85.7 \%(12 / 14)$ for ELISA A and $71.4 \%$ (10/14) for ELISA B. The BTM test correctly classified inside the $>0$ to $<10 \%$ group $36.8(21 / 57)$ and $43.9 \%$ $(25 / 57)$ of the herds with ELISA A and B, respectively (Table 3 ). Considering jointly positive and highly posi- 
Table 1. Mean corrected optical densities (as determined by antibody ELISA) recorded in bulk tank milk (BTM) samples by within-herd seroprevalence group

\begin{tabular}{llrrr}
\hline & & & \multicolumn{2}{c}{ CI (95\%) } \\
\cline { 4 - 4 } ELISA $^{1}$ & BTM result & OD $^{2}$ (mean) & Lower limit & Upper limit \\
\hline ELISA A & Negative & 3.8636 & 2.9699 & 4.7574 \\
& Positive & 10.2982 & 7.0301 & 13.5664 \\
& High positive & 27.0714 & 13.7859 & 40.3570 \\
& Total & 7.2709 & 5.6873 & 8.8546 \\
ELISA B & Negative & -1.4924 & -1.7806 & -1.2042 \\
& Positive & -0.7493 & -1.0976 & -0.4010 \\
& High positive & 5.2500 & -1.0757 & 11.5757 \\
& Total & -0.8188 & -1.3152 & -0.3223 \\
\hline
\end{tabular}

${ }^{1}$ ELISA A $=$ ELISA Idexx Paratuberculosis: Idexx Paratuberculosis Screening Ab Test, Westbrook, ME; ELISA B: Parachek 2: Mycobacterium paratuberculosis test kit Parachek 2, Prionics AG, Zurich, Switzerland.

${ }^{2} \mathrm{OD}=$ corrected optical density.

tive herds ( $>0 \%$ seropositive animals), the BTM test Se values were $76.1 \%(54 / 71)$ for ELISA A and $78.9 \%$ $(56 / 71)$ for ELISA B.

The $\mathrm{Sp}$ when testing negative herds (0\% seroprevalence) was $70.5 \%(93 / 132)$ for ELISA A and 53\% (70/132) for ELISA B (Table 3). Considering the prevalence conditions of the studied population, the negative predictive values (NPV) were $84.5 \%$ (93/110) for ELISA A and $82.4 \%(70 / 85)$ for ELISA B. In a quantitative approach, the $\rho$ values observed when comparing the antibody levels in BTM and the within-prevalence were moderate (0.541 and 0.391 for ELISA A and B, respectively) with a correlation of 0.766 between the BTM antibody levels recorded by both ELISA.

One of the advantages of many years of paratuberculosis research and control in northwestern Spain is the presence of a large number of negative herds, and several herds with different within-herd prevalence. As far as the laboratory tests are concerned, the program was based on the use of antibody ELISA to test serum samples from animals older than 24 mo to determine the serological profile of the herds and to identify cows most likely to shed the organism; fecal samples of the positives were collected and these were tested for the presence of MAP by PCR. In addition, testing of purchased animals is compulsory for farms that have joined the program.

Our study intended to provide a logical validation for the use of bulk milk ELISA-corrected OD to scale the risk of MAP infection in the lactating herd. The lactating group, although not including the whole herd, is the most numerous in the set of adult animals, which are those that most probably would have developed serological responses to MAP.

According to van Weering et al., (2007), the relative Se of testing individual milk samples as compared with serum samples was high (between 87 and 96\%). The present study indicated that the Se of testing BTM samples to detect positive or highly positive herds showed lower values, ranging from $76.1 \%$ for ELISA A to $78.9 \%$ for ELISA B; to detect only high-prevalence levels $(\geq 10 \%)$, the Se increased to 85.7 and $71.4 \%$. The Sp to detect herds with no seropositive animals was lower than the Se and, even some herds with $0 \%$ seroprevalence were classified as highly positive when using BTM samples, especially by ELISA B.

The ELISA Se is a direct function of the distribution of the infection stages in the test population. False-

Table 2. Threshold values of transformed optical density from different ELISA in bulk tank milk samples for the established herd seroprevalence level ${ }^{1}$

\begin{tabular}{lcc}
\hline ELISA & Prevalence & Interval \\
\hline ELISA A & $0 \%$ & $\leq 3$ \\
(interval in: sample/positive index) & $>0-<10 \%$ & $>3-<10.5$ \\
& $\geq 10 \%$ & $\geq 10.5$ \\
ELISA B & $0 \%$ & $\leq-1.44$ \\
(interval in: sample/positive index) & $>0-<10 \%$ & $\geq(-1.44)-<(0.5)$ \\
& $\geq 10 \%$ & $\geq 0.5$
\end{tabular}

${ }^{1}$ Prevalence $=$ percentage of seropositive animals; ELISA A = ELISA Idexx Paratuberculosis: Idexx Paratuberculosis Screening Ab Test, Westbrook, ME; ELISA B = Parachek 2: Mycobacterium paratuberculosis test kit Parachek 2, Prionics AG, Zurich, Switzerland. 
Table 3. Cross-classification of the results of an antibody ELISA from samples of bulk-tank milk (BTM) and within-herd prevalences from serum samples

\begin{tabular}{|c|c|c|c|c|c|c|c|c|}
\hline \multirow[b]{3}{*}{ BTM result } & \multicolumn{8}{|c|}{ Within-herd prevalence $^{1}$} \\
\hline & \multicolumn{3}{|c|}{ ELISA A } & \multicolumn{4}{|c|}{ ELISA B } & \multirow[b]{2}{*}{ Total } \\
\hline & $\begin{array}{c}\text { Negative } \\
(0 \%)\end{array}$ & $\begin{array}{l}\text { Positive } \\
(\geq 0-10 \%)\end{array}$ & $\begin{array}{c}\text { High } \\
\text { positive } \\
(\geq 10 \%)\end{array}$ & Total & $\begin{array}{c}\text { Negative } \\
(0 \%)\end{array}$ & $\begin{array}{l}\text { Positive } \\
(\geq 0-10 \%)\end{array}$ & $\begin{array}{c}\text { High } \\
\text { positive } \\
(\geq 10 \%)\end{array}$ & \\
\hline Negative & 93 & 17 & 0 & 110 & 70 & 13 & 2 & 85 \\
\hline Positive & 32 & 21 & 2 & 55 & 35 & 25 & 2 & 62 \\
\hline High positive & 7 & 19 & 12 & 38 & 27 & 19 & 10 & 56 \\
\hline Total & 132 & 57 & 14 & 203 & 132 & 57 & 14 & 203 \\
\hline
\end{tabular}

${ }^{1}$ ELISA A = ELISA Idexx Paratuberculosis: Idexx Paratuberculosis Screening Ab Test, Westbrook, ME;

ELISA B = Parachek 2: Mycobacterium paratuberculosis test kit Parachek 2, Prionics AG, Zurich, Switzerland.

negative results are more likely to occur in the initial stages of the infection, but this should not affect the relation between BTM and cow-level reactions because lack of antibodies would affect both the BTM and the individual cow samples. (Nielsen and Toft, 2014). Likewise, factors that affect $\mathrm{Sp}$, such as exposure to mycobacteria other than MAP, would essentially be likely to contribute to both within-herd seroprevalence as well as BTM test reaction (Nielsen and Toft, 2014).

Discrepancies when comparing within herd seroprevalences and BTM antibody levels could be explained by varying contributions of individual cows to the BTM, different milk yields of antibody-negative and -positive cows, or could even reflect the incorporation of new seropositive animals in farms that were less committed to controlling paratuberculosis. Therefore, these factors should be considered when interpreting BTM antibody ELISA results, especially if the farm in question is small. In the study population, the mean herd size was 51 cows but with a significant deviation, as mentioned. This implies the presence of farms of small size may have some effect on the results. The BTM antibody ELISA has been successfully used in control programs for other endemic diseases of cattle, such as BVD or IBR, to know the level of exposure of the herd to these viruses (Nylin et al., 1999; Eiras et al., 2012); in any case, the dynamics of infection, the generation of antibodies, and the antibody excretion patterns through milk, are very different from that which occurs after MAP infection.

These results of the present paper are partially in agreement with other studies. Nielsen et al. (2000) concluded that the ELISA was not sufficient to provide a tool for MAP surveillance. Nevertheless, it can be used with some modifications for detection of high-prevalence herds but not herds with a very low prevalence. Whereas bulk milk testing would be a useful screening test for regional or national programs, the NPV would be particularly important (van Weering et al., 2007). Considering the prevalence values in the study population, the results indicated fair to good NPV (from $82.4 \%$ for ELISA B to $84.5 \%$ for ELISA A); assuming all other factors remain constant, the NPV would increase with decreasing prevalence.

Our study showed poor to fair agreement when comparing the level of antibodies in BTM samples and the within-herd seroprevalence The Se values of BTM test to detect positive ( $>0 \%$ seroprevalence) or highly positive herds $(\geq 10 \%$ seroprevalence) reach a maximum of 78.9 and $85.7 \%$, respectively; whereas the $\mathrm{Sp}$ to values to detect negative herds were still lower.

\section{ACKNOWLEDGMENTS}

The authors thank the Xunta de Galicia (Galicia, Spain), 2016 GPC GI-1948 for financial support.

\section{REFERENCES}

Diéguez, F. J., E. Yus, M. L. Sanjuán, M. J. Vilar, and I. Arnaiz. 2008. Monitoring bovine viral diarrhea virus (BVDV) infection status in dairy herds. Pesqui. Vet. Bras. 28:588-592.

Eiras, C., I. Arnaiz, M. L. Sanjuán, E. Yus, and F. J. Diéguez. 2012. Bovine viral diarrhea virus: Correlation between herd seroprevalence and bulk tank milk antibody levels using 2 commercial immunoassays. J. Vet. Diagn. Invest. 24:549-553.

Khol, J. L., M. Wassertheurer, E. Sodoma, S. Revilla-Fernández, J. Damoser, E. Österreicher, M. Dünser, U. Kleb, and W. Baumgartner. 2013. Long-term detection of Mycobacterium avium subspecies paratuberculosis in individual and bulk tank milk from a dairy herd with a low prevalence of Johne's disease. J. Dairy Sci. 96:35173524 .

Lavers, C. J., H. W. Barkema, I. R. Dohoo, S. L. B. McKenna, and G. P. Keefe. 2014. Evaluation of milk ELISA for detection of $M y-$ cobacterium avium subspecies paratuberculosis in dairy herds and association within-herd prevalence. J. Dairy Sci. 97:299-309.

Lombard, J. E., T. M. Byrem, B. A. Wagner, and B. J. McCluskey. 2006. Comparison of milk and serum enzyme-linked immunosorbent assays for diagnosis of Mycobacterium avium subspecies paratuberculosis infection in dairy cattle. J. Vet. Diagn. Invest. 18:448-458. 
Ministerio de Agricultura y Pesca, Alimentación y Medio Ambiente (MAPAMA). 2015. Estadísticas Agrarias: Ganadería. Accessed Dec. 19, 2016. http://www.mapama.gob.es/es/estadistica/temas/ estadisticas-agrarias/ganaderia/default.aspx.

Nielsen, S. S., S. M. Thamsborg, H. Houe, and V. Bitsch. 2000. Bulktank milk ELISA antibodies for estimating the prevalence of paratuberculosis in Danish dairy herds. Prev. Vet. Med. 44:1-7.

Nielsen, S. S., and N. Toft. 2014. Bulk tank milk ELISA for detection of antibodies to Mycobacterium avium ssp. paratuberculosis: Correlation between repeated tests and within-herd antibody-prevalence. Prev. Vet. Med. 113:96-102.

Nylin, B., U. Strøger, and L. Rønsholt. 1999. A retrospective evaluation of a bovine herpesvirus-1 (BHV-1) antibody ELISA on bulktank milk samples for classification of the BHV-1 status of Danish dairy herds. Prev. Vet. Med. 47:91-105. van Weering, H., G. van Schaik, A. van der Meulen, M. Waal, P. Franken, and K. van Maanen. 2007. Diagnostic performance of the Pourquier ELISA for detection of antibodies against Mycobacterium avium subspecies paratuberculosis in individual milk and bulk milk samples of dairy herds. Vet. Microbiol. 125:49-58.

Warnick, L. D., L. R. Nielsen, J. Nielsen, and M. Greiner. 2006. Simulation model estimates of test accuracy and predictive values for the Danish Salmonella surveillance program in dairy herds. Prev. Vet. Med. 77:284-303.

Wilson, D. J., K. Rood, P. Biswas, and T. M. Byrem. 2010. Herd-level prevalence of Johne's disease in Utah and adjacent areas of the Intermountain West as detected by a bulk-tank milk surveillance project. J. Dairy Sci. 93:5792-5797. 